9-1996

\title{
Globally Optimal Periodic Robot Joint Trajectories
}

Daniel J. Simon

Cleveland State University, d.j.simon@csuohio.edu

Follow this and additional works at: https://engagedscholarship.csuohio.edu/enece_facpub

Part of the Robotics Commons

How does access to this work benefit you? Let us know!

Publisher's Statement

NOTICE: this is the author's version of a work that was accepted for publication in Journal of the Franklin Institute. Changes resulting from the publishing process, such as peer review, editing, corrections, structural formatting, and other quality control mechanisms may not be reflected in this document. Changes may have been made to this work since it was submitted for publication. A definitive version was subsequently published in Journal of the Franklin Institute, 333, 5, (09-01-1996); dx.doi.org/10.1016/0016-0032(96)00044-0

\section{Original Citation}

D. Simon. (1996). Globally Optimal Periodic Robot Joint Trajectories. Journal of the Franklin Institute, 333(5), pp. 659-668, doi: dx.doi.org/10.1016/0016-0032(96)00044-0.

\section{Repository Citation}

Simon, Daniel J., "Globally Optimal Periodic Robot Joint Trajectories" (1996). Electrical Engineering \& Computer Science Faculty Publications. 134.

https://engagedscholarship.csuohio.edu/enece_facpub/134

This Article is brought to you for free and open access by the Electrical Engineering \& Computer Science Department at EngagedScholarship@CSU. It has been accepted for inclusion in Electrical Engineering \& Computer Science Faculty Publications by an authorized administrator of EngagedScholarship@CSU. For more information, please contact library.es@csuohio.edu. 


\section{Globally Optimal Periodic Robot Foint Trajectories}

by DAN SIMON

TRW Test Laboratory, 4051 N. Higley Road, Mesa, AZ 85215, U.S.A.

\section{Introduction}

The industrial robot is a highly nonlinear, coupled multivariable system with nonlinear constraints. For this reason, robot control algorithms are often divided into two stages: path planning and path tracking. A conceptually simple approach to the path planning problem is to generate a joint-space trajectory based on interpolation of a sequence of desired joint angles. This approach ignores most of the dynamics of the robot. So the resultant trajectories do not take full advantage of the robot's capabilities, but are computationally easy to obtain. In this approach, a number of knot points are chosen along the desired Cartesian path. The number of knots chosen is a tradeoff between exactness and computational expense. The Cartesian knots are then mapped into joint knots using inverse kinematics. Finally, an analytic interpolating curve is fitted to the joint knots. This curve provides the path tracker with joint angles and derivatives at the controller rate.

The most popular type of interpolation is algebraic splines $(\mathbf{1 , 2})$. An $n$ th-order algebraic spline consists of piecewise-continuous $n$ th-order algebraic polynomials which have continuous derivatives up to order $(n-2)$ or less (depending on the details of the formulation). Higher order splines result in continuity of higher order derivatives, which reduces wear and tear on the robot, but this is at the expense of large oscillations of the trajectory.

Polynomials with order as low as five (e.g. quartic splines) can overshoot extreme knots by as much as 60 degrees (3). A recent development is the use of trigonometric polynomials to efficiently generate joint trajectories with little overshoot but continuous velocity, acceleration, and jerk $(4,5)$. Trigonometric polynomials can be normalized in 
time so as to be very smooth if their order is moderate (6). If piecewise continuous trigonometric polynomials are pieced together, the computational expense is low, and each polynomial is of low order, preventing oscillations between knots.

In many applications, a robot is required to perform a repetitive task. Some examples include assembly line work, welding, and spray painting. In this paper, Schoenberg splines are used to generate globally optimal periodic joint trajectories. Schoenberg splines are bilinear functions of algebraic and trigonometric polynomials. The optimality criterion is a combination of the integral of the square of joint velocity and jerk. Of course, the robot has to begin and end its motion at some finite time. So we constrain the joint velocity and acceleration at the start of each periodic to be zero, so that the robot can begin and end its motion smoothly. The joint trajectories have continuous derivatives up to the fourth order except at the start of each period. At the start of each period, the derivatives are continuous up to the second order.

Section II summarizes the main results of interpolation with Schoenberg splines, and discusses their extension to hermite interpolation (i.e. interpolation of knot derivatives as well as knot values). Section III discusses the application of Schoenberg splines to the robot path planning problem. Section IV presents a numerical example, and Section $\mathrm{V}$ presents some concluding remarks.

\section{Schoenberg Splines}

The term trigonometric spline was first introduced by Schoenberg (7). But since then other definitions have appeared in the literature $(\mathbf{8 , 9})$. So the term is not well defined. Trigonometric splines have recently been applied to robot path planning $(\mathbf{4 , 5})$. But those trigonometric splines are different than those Schoenberg referred to as trigonometric splines. Therefore, Schoenberg's trigonometric splines will be referred to in this paper as Schoenberg splines. In this section, the definition and some of the properties of these functions will be given.

\subsection{Problem statement and main results}

This subsection summarizes some of the results of Schoenberg's original paper. Proofs for the theorems in this subsection can be found in Schoenberg (7).

\section{Problem statement}

Within the class of functions $f(t)$ of period $2 \pi$ which satisfy the interpolation conditions $f\left(t_{i}\right)=y_{i}(i=1, \ldots, n)$, where $0 \leqslant t_{1}<t_{2}<\cdots<t_{n}<2 \pi$, we seek a solution to the problem

$$
\int_{0}^{2 \pi}\left[\Delta_{m} f(t)\right]^{2} d t=\text { minimum }
$$

where $n \geqslant(2 m+1)$, and the operator $\Delta_{m}$ is given by

$$
\Delta_{m}=D\left(D^{2}+1^{2}\right)\left(D^{2}+2^{2}\right) \ldots\left(D^{2}+m^{2}\right)
$$

where $D$ is the differential operator. 


\section{Definition 1}

A Schoenberg spline of order $m$, having the distinct knots $\left(t_{1}, \ldots, t_{n}\right) \in(0,2 \pi)$, is a function $S(t)$ which has the following properties.

1. $S(t)$ has period $2 \pi$ and is $4 m$ times continuously differentiable.

2. In each of the $n$ closed arcs $\left[t_{j}, t_{j}+1\right], \Delta_{m}^{2} S(t)=0$. This property can alternatively be characterized by stating that in each of the $n$ closed arcs $\left[t_{j}, t_{j}+1\right]$,

$$
S(t) \in \operatorname{Span}[1, \cos r t, \sin r t, t, t \cos r t, t \sin r t] \quad(r=1, \ldots, m) .
$$

\section{Theorem I}

If $n \geqslant(2 m+1)$ there is a unique Schoenberg spline $S(t)$ of order $m$ which satisfies $S\left(t_{i}\right)=y_{i}(i=1, \ldots, n)$. In addition, the $m$ th-order Schoenberg spline $S(t)$ satisfies the minimum property of Eq. (1).

\section{Theorem II}

The $m$ th-order Schoenberg spline $S(t)$ satisfying $S\left(t_{i}\right)=y_{i}(i=1, \ldots, n)$ is represented uniquely by

$$
S(t)=\sum_{j=1}^{n} c_{j} \psi_{m}\left(t-t_{j}\right) \tau_{m}(t)
$$

where $\tau_{m}(t)$ is an $m$ th-order trigonometric polynomial, and $\psi_{m}(t)$ is given by

$$
\begin{aligned}
\psi_{m}(t) & =\sum_{k=m+1}^{\infty} \frac{\cos (k t)}{k^{2}\left(1^{2}-k^{2}\right)^{2} \ldots\left(m^{2}-k^{2}\right)^{2}} \\
& =t(2 \pi-t) C_{m}(t)+(\pi-t) S_{m}(t) 0 \leqslant t \leqslant 2 \pi .
\end{aligned}
$$

$C_{m}(t)$ is an $m$ th-order cosine polynomial, $S_{m}(t)$ is an $m$ th-order sine polynomial, and $\psi_{m}(t)$ is extended beyond the interval $[0,2 \pi]$ so as to have a period of $2 \pi$. The coefficients of $C_{m}(t)$ and $S_{m}(t)$ satisfy the equality

$$
2 t C_{m}(t)+S_{m}(t)=\frac{-1}{2(4 m+1) !} t^{4 m+1}+\text { HOT }(\text { higher order terms })
$$

and the coefficients $c_{j}$ satisfy the equality

$$
\sum_{j=1}^{n} c_{j} \phi\left(t-t_{j}\right)=0
$$

where $\phi(t)$ is given by

$$
\phi(t)=\frac{2^{m}}{(2 m) !}(1-\cos t)^{m}
$$

Note that the left-hand sides of Eqns (6) and (7) are each $m$ th-order trigonometric functions. Therefore Eqns (6) and (7) each give $(2 m+1)$ constraints. The interpolation conditions $S\left(t_{i}\right)=y_{i}$ give $n$ constraints. These $(n+4 m+2)$ constraints determine the $n$ coefficients $c_{j}$, the $(2 m+1)$ coefficients of $\tau_{m}(t)$, the $(m+1)$ coefficients of $C_{m}(t)$, and the 
$m$ coefficients of $S_{m}(t)$. These $(n+4 m+2)$ coefficients in turn completely specify the Schoenberg spline $S(t)$ [see Eq. (4)].

\subsection{Extension to multiple knots}

The theory of the preceding subsection can be extended in a straightforward manner to the case of coincident knots. This allows the spline and its derivatives to be constrained at given knots.

Assume that the first knot has multiplicity $r$. That is, $t_{1}=t_{2}=\cdots=t_{r}<t_{r+1}<$ $\cdots<t_{n}$. Then the order of continuity of $S(t)$ at $t=t_{1}$ drops from $4 m$ to $(4 m-r+1)$. The range of $r$ is restricted to $1<r<(2 m+1)$. In this case, Eq. (4) is generalized to

$$
S(t)=\sum_{j=0}^{r-1} c_{1}^{j} \psi_{m}^{(j)}\left(t-t_{1}\right)+\sum_{j=r+1}^{n} c_{j} \psi_{m}\left(t-t_{j}\right)+\tau_{m}(t) .
$$

$S(t)$ is now required to satisfy the interpolation conditions

$$
\begin{gathered}
S^{(j)}\left(t_{1}\right)=y_{1}^{(j)} \quad(j=1, \ldots, r-1) \\
S\left(t_{k}\right)=y_{k} \quad(k=1, \ldots, n) .
\end{gathered}
$$

The constraints of the $c$ coefficients [see Eq. (7)] become

$$
\sum_{j=0}^{r-1} c_{1}^{(j)} \phi^{(j)}\left(t-t_{1}\right)+\sum_{j=r+1}^{n} c_{j} \phi\left(t-t_{j}\right)=0 .
$$

The number of constraints used to determine the coefficients of $S(t)$ has increased by $(r-1)$ [see Eq. (10)]. These additional constraints are sufficient to determine the $(r-1)$ additional coefficients $c_{1}^{(j)}(j=1, \ldots, r-1)$. Nothing else in the previous subsection changes.

\section{Application to Robot Path Planning}

Now the theory of the previous section will be applied to the robot path planning problem. Consider a periodic joint trajectory $h(t)$ which is required to pass through knots $\left(h_{1}, \ldots, h_{n}\right)$ at times $\left(t_{1}, \ldots, t_{n}\right)$ where $t_{1}=0<t_{2}<\cdots<t_{n}$. Such a trajectory would be specified, for example, if a robot was required to repetitively perform the same assembly task many times. The joint trajectory is required to have zero velocity and acceleration at time $t_{1}$. This ensures that at the beginning of the day, the robot motion begins smoothly, and at the end of the day, the robot comes to a smooth stop. The first-order Schoenberg spline satisfying these interpolation conditions will give the joint trajectory which, among all $2 \pi$-periodic functions $f(t)$ satisfying the desired interpolation conditions, minimizes

$$
\int_{0}^{2 \pi}\left\{\left[f^{\prime \prime \prime}(t)\right]+\left[f^{\prime}(t)\right]\right\}^{2} \mathrm{~d} t
$$

Of course, it is quite unlikely that the robot motion is required to have a period of $2 \pi$. So the trajectory $h(t)$ will need to be scaled so as to give a trajectory $g(t)$ with the same shape as $h(t)$, but with the desired period $T$. 


$$
g(t)=h(2 \pi t / T)
$$

Then the real-time trajectory will satisfy the following optimality criterion. Among all functions $f(t)$ of period $T$ which satisfy the desired interpolation conditions, $g(t)$ minimizes

$$
\int_{0}^{T}\left\{\left[(2 \pi / T) f^{\prime \prime \prime}(t)\right]+\left[f^{\prime}(t)\right]\right\}^{2} \mathrm{~d} t
$$

This shows that for a trajectory with $T>2 \pi$, the velocity will be given a greater weight than the jerk. For a trajectory with $T<2 \pi$, the jerk will be given a greater weight. Equation (9) (with a slight change of notation) gives

$$
h(t)=\sum_{j=0}^{2} c_{1}^{(j)} \psi^{(j)}(t)+\sum_{j=2}^{n} c_{j} \psi\left(t-t_{j}\right)+A \cos t+B \sin t+C .
$$

Equation (5) shows that the $2 \pi$-periodic function $\psi(t)$ is given by

$$
\begin{gathered}
\psi(t)=t(2 \pi-t) C_{1}(t)+(\pi-t) S_{1}(t) \quad 0 \leqslant t \leqslant 2 \pi \\
C_{1}(t)=a_{0}+a_{1} \cos t \\
S_{1}(t)=b_{1} \sin t
\end{gathered}
$$

Equation (6) gives

$$
2 t C_{1}(t)+S_{1}(t)=-t^{5} / 240+\text { HOT }
$$

which yields [via Taylor series expansions of $C_{1}(t)$ and $S_{1}(t)$ ]

$$
\begin{gathered}
a_{0}=-1 / 14 \\
a_{1}=-1 / 28 \\
b_{1}=3 / 14 .
\end{gathered}
$$

Equation (12) shows that the $c$ coefficients satisfy

$$
\sum_{j=0}^{2} c_{1}^{(j)} \phi^{(j)}(t)+\sum_{j=2}^{n} c_{j} \phi\left(t-t_{j}\right)=0
$$

where $\phi(t)$ is given by

$$
\phi(t)=1-\cos t .
$$

Note that Eq. (24) actually gives three constraints since it is a linear combination of the linearly independent functions $[1, \cos t, \sin t]$. By using Eq. (25), we can expand Eq. (24) as

$$
\left[c_{1}^{(0)}+\sum_{j=2}^{n} c_{j}\right]+\left[-c_{1}^{(0)}+c_{1}^{(2)}-\sum_{j=2}^{n} c_{j} \cos t_{j}\right] \cos t+\left[c_{1}^{(1)}-\sum_{j=2}^{n} c_{j} \sin t_{j}\right] \sin t=0 .
$$


TABLE I

Cartesian and joint-space knots

\begin{tabular}{lcccc}
\hline \multirow{2}{*}{ Knot number } & \multicolumn{2}{c}{ Cartesian knots } & \multicolumn{2}{c}{ Joint-space knots } \\
& $X$ & $Y$ & $\theta_{1}$ & \multicolumn{1}{c}{$\theta_{2}$} \\
\hline 1 & 0.8 & 0.0 & 36.9 & -73.7 \\
2 & 0.2 & 0.3 & 125.2 & -137.7 \\
3 & 0.0 & 0.8 & 126.9 & -73.7 \\
4 & -0.2 & 0.3 & 192.6 & -137.7 \\
5 & -0.5 & 0.3 & 203.4 & -108.87 \\
6 & -0.2 & 0.3 & 192.6 & -137.7 \\
7 & 0.0 & 0.8 & 126.9 & -73.7 \\
8 & 0.2 & 0.3 & 125.2 & -137.7 \\
\hline
\end{tabular}

Note from Eq. (16) that the optimal $2 \pi$-periodic joint trajectory $h(t)$ depends on the $(n+5)$ coefficients $c_{1}^{(j)}(j=0,1,2), c_{j}(j=2, \ldots, n), A, B$, and $C$. The $(n+5)$ constraints which uniquely determine these coefficients are $h^{\prime}\left(t_{1}\right)=h^{\prime \prime}\left(t_{1}\right)=0, h\left(t_{j}\right)=h_{j}(j=$ $1, \ldots, n)$, and the three constraints of Eq. (26). The solvability of this system of linear equations is guaranteed by the existence and uniqueness of the Schoenberg spline [see Section II].

\section{Numerical Example}

Software has been written to implement Schoenberg spline trajectory generation for a simple two-link manipulator. Each link is 0.5 meters in length. The first joint angle $\theta_{1}$ is taken as the angle from the horizontal positive $x$-direction to the first link. The second joint angle $\theta_{2}$ is taken as the angle from the outward direction of the first link to the second link. Both joint angles are measured in the counterclockwise direction.

Eight Cartesian knots equally spaced in time are specified to represent what might be a typical periodic task for an industrial robot under the constraint of obstacle avoidance. One period of the robot motion is specified to be $40 \mathrm{~s}$. After the robot reaches the eighth knot, it returns to its starting position (the first knot) and repeats its performance.

The Cartesian and joint-space knots are given in Table $I$ in units of meters and degrees. The joint space trajectories and derivatives are shown for one period in Figs $1-4$. Figure 5 shows the knot locations, obstacle locations, and the Cartesian path resulting from the use of joint-space Schoenberg splines. The robot trajectory is symmetric with respect to the midpoint of its period. So during the second half of its period, the robot retraces the route taken during the first half.

Note that the velocity and acceleration are continuous over the entire path, and are zero at the first knot, allowing for a smooth starting and stopping motion. Note that the jerk is continuous on the interior of the trajectory, but discontinuous at the start of each period. This is due to the fact that velocity and acceleration are constrained at the start of each period. Also note the relatively low magnitudes of the joint velocities 


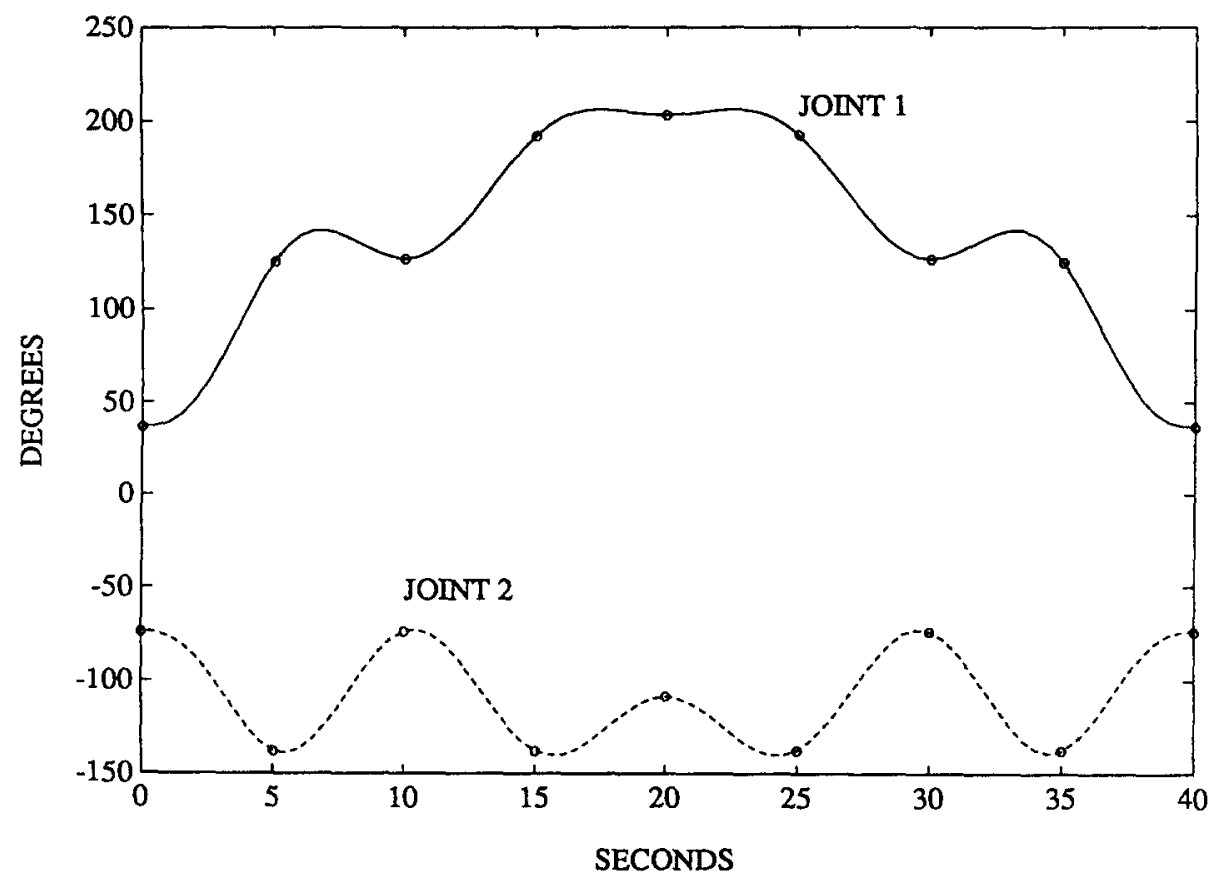

FIG. 1. Joint trajectories.

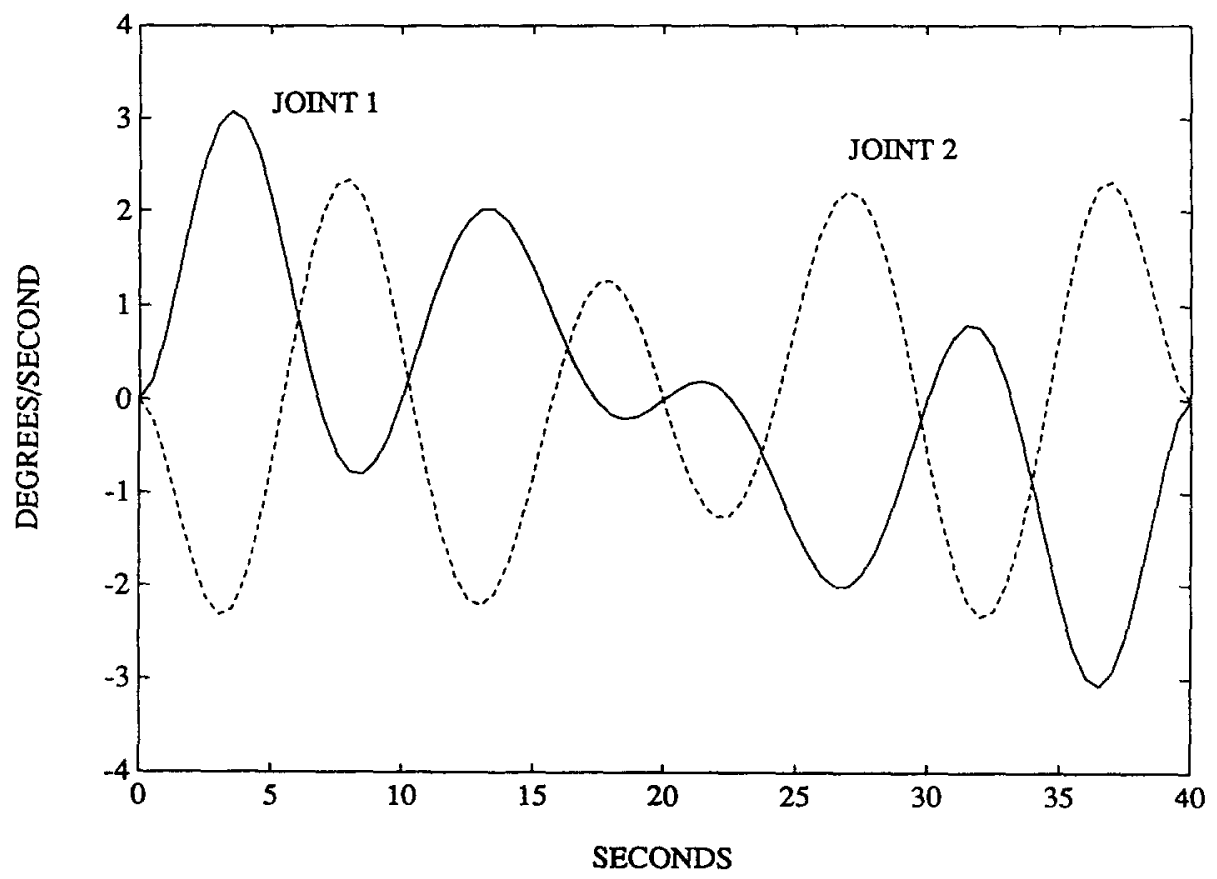

FIG. 2. Joint velocities. 


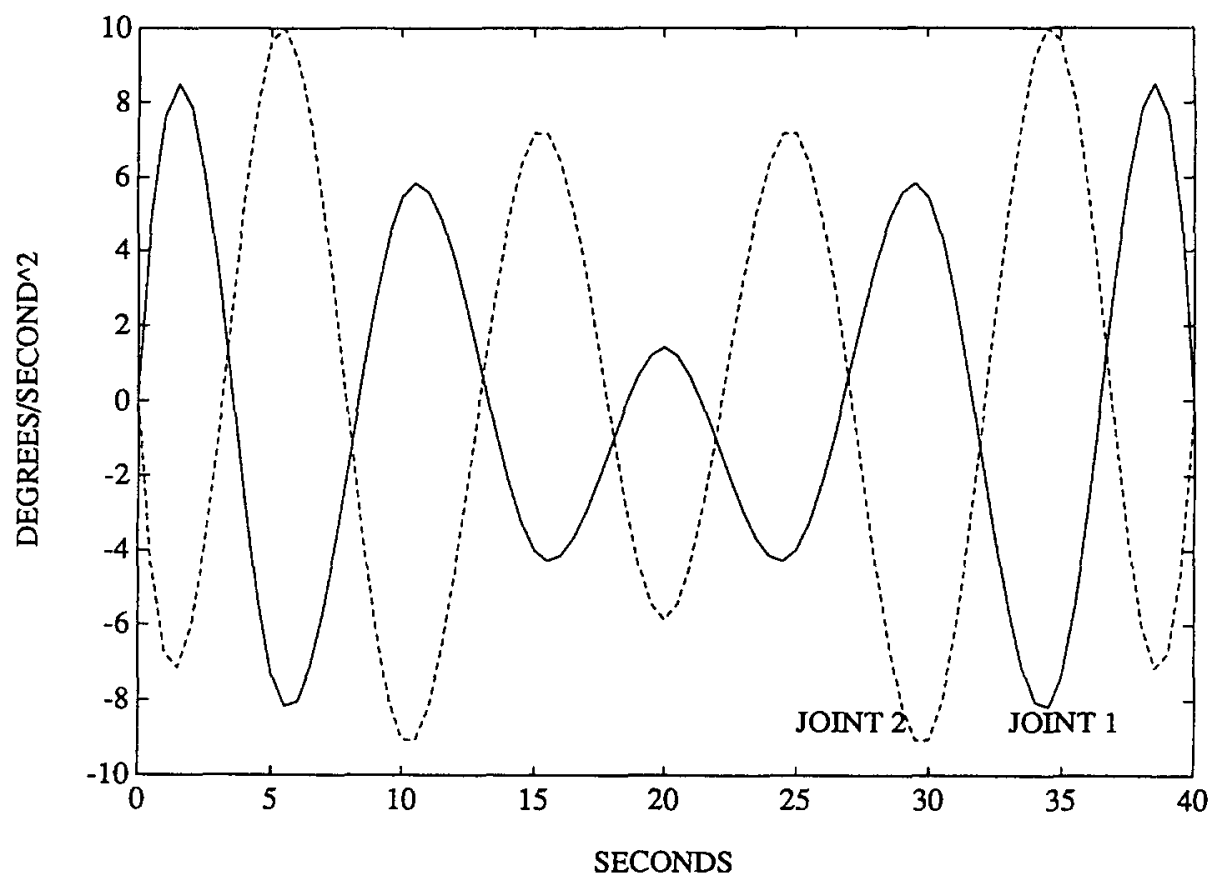

FIG. 3. Joint accelerations.

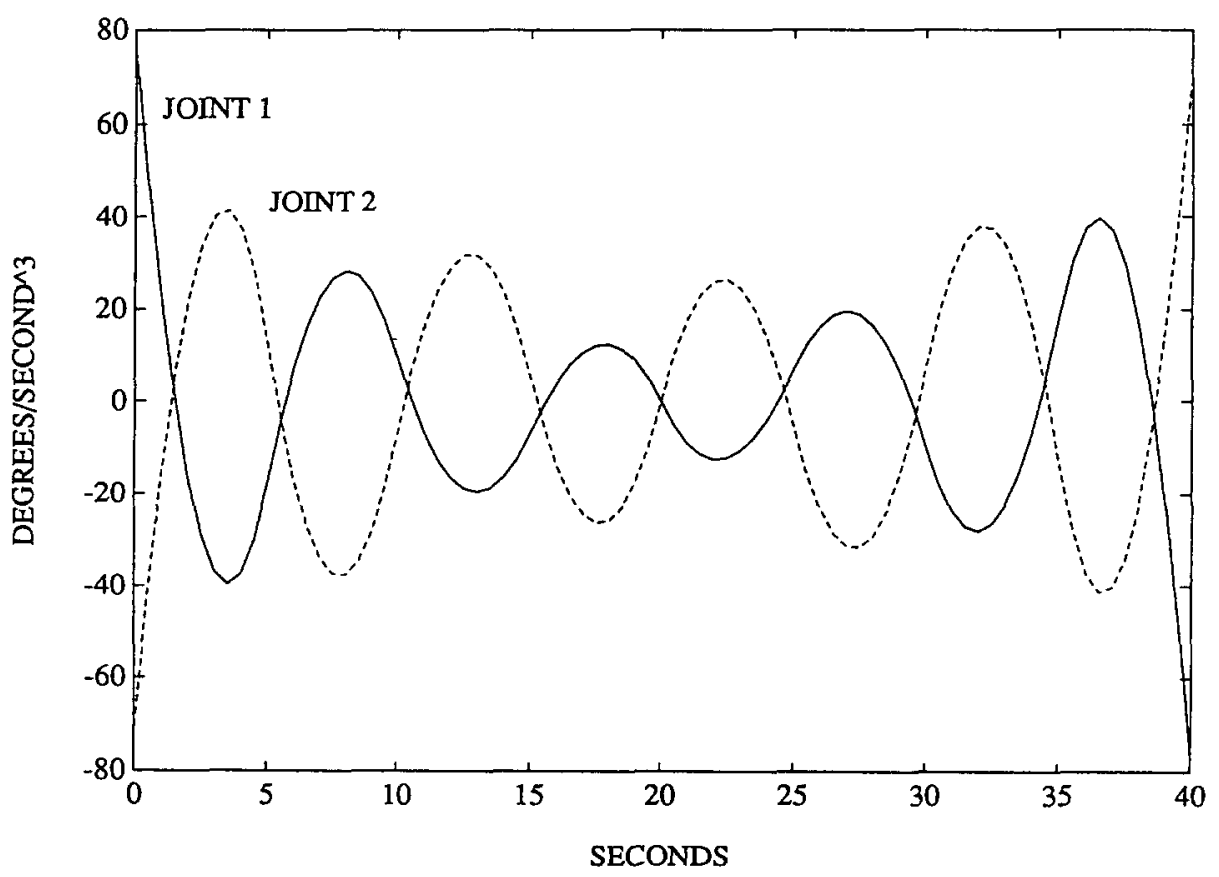

FIG. 4. Joint jerks. 


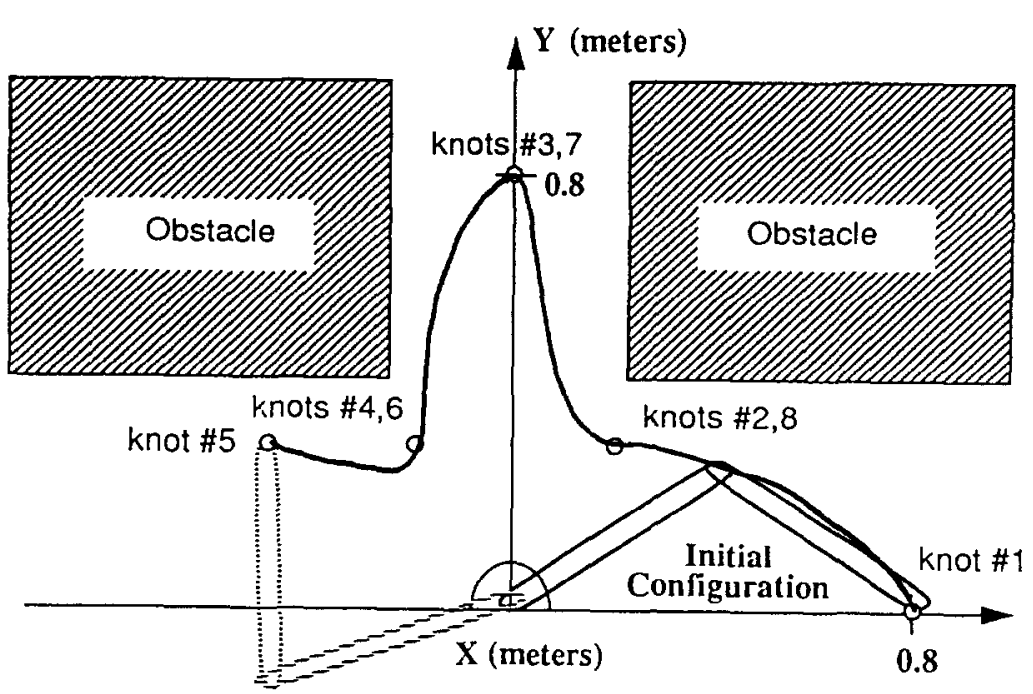

FIG. 5. Cartesian path.

as compared to the joint jerks. This is due to the fact that $T=40 \gg 2 \pi$, so the velocity is given a much greater weight in the objective function that the jerk [see Eq. (15)]. If the resultant joint derivatives violate the limits of the robot, the trajectory can simply be scaled in time so that the limits are not violated.

\section{Conclusion}

The ability to plan joint-space trajectories through a sequence of specified knots is an important aspect of trajectory planning. The knots can be chosen to satisfy the joint limit constraints and avoid obstacles. The use of Schoenberg splines presented in this paper is attractive in that globally optimal joint trajectories can be generated for periodic robot tasks. Although the objective function does not consider the dynamics of the robot, this global optimality is a feature found in few, if any, other trajectory generation methods. The resultant trajectory has continuous derivatives up to the second order everywhere along the trajectory. In addition, the trajectory has continuous jerk everywhere except at the beginning of each period. The trajectory could thus be used as a starting point for a trajectory generator which takes into account such things as robot dynamics, joint angle restrictions, speed restrictions, etc. The generation of Schoenberg trajectories is computationally reasonable, requiring the solution of $(n+5)$ simultaneous linear equations, where $n$ is the number of knots in one period.

\section{References}

(1) C. Lin and P. Chang, "Joint trajectories of mechanical manipulators for Cartesian path approximation", IEEE Trans. on Systems, Man, and Cybernetics, Vol. SMC-13, pp. 1094 $1102,1983$.

(2) S. Chand and K. Doty, "On-line polynomial trajectories for robot manipulators", Int. J. Robot. Res. Vol. 4, pp. 38-48, 1985. 
(3) S. Thompson and R. Patel, "Formulation of joint trajectories for industrial robots using Bsplines", IEEE Trans. on Industrial Electronics, Vol. IE-34, pp. 192-199, 1987.

(4) D. Simon and C. Isik, "The generation and optimization of trigonometric joint trajectories for robotic manipulators", in "Proc. American Control Conf.", pp. 2027-2032, Boston, MA, 1991.

(5) D. Simon and C. Isik, "Optimal trigonometric robot joint trajectories", Robotica, Vol. 9, pp. 379-386, 1991.

(6) D. Simon, "A unified approach to robot path planning using trigonometric splines", Ph.D. Dissertation, Syracuse University, Department of Electrical Engineering, Syracuse, NY, 1991.

(7) I. Schoenberg, "On trigonometric spline interpolation", J. Math. Mechan. Vol. 13, pp. 795$825,1964$.

(8) T. Lyche and R. Winther, "A stable recurrence relation for trigonometric B-splines", $J$. Approx. Theory, Vol. 25, pp. 266-279, 1979.

(9) P. Koch, "Error Bounds for Interpolation by Fourth Order Trigonometric Splines", in "Approximation Theory and Spline Functions", (Edited by S. Singh, J. Burry, and B. Watson), pp. 349-360, D. Reidel Publishing Company, Dordrecht, Holland, 1984. 